\title{
ATM Direct Charging Reform: the Effect of Independent Deployers on Welfare
}

\author{
Jocelyn Donze*and Isabelle Dubec ${ }^{\dagger}$
}

July 5,2011

\begin{abstract}
In Australia, interchange fees on shared ATM transactions have been removed since March 2009 and replaced by usage fees directly set and received by ATM owners. We develop a model to study how the entry of independent ATM deployers (IADs) affects welfare under the "direct charging scheme". We show that paradoxically, IAD entry benefits banks. Entry also benefits consumers when they strongly value the associated growth of the ATM network.

JEL classification: L1, G2
\end{abstract}

*Toulouse School of Economics (GREMAQ); jocelyn.donze@tse-fr.eu

†Toulouse School of Economics (GREMAQ); isabelle.dubec@tse-fr.eu 


\section{Introduction}

The Australian ATM market is characterized by two features (Reserve Bank of Australia, 2000 \& 2009; Filipovski and Flood, 2010). First of all, since March 2009, interchange fees and foreign fees have been removed and replaced by a pricing scheme where ATM owners directly charge a fee to any cardholder who uses their ATMs. ${ }^{1}$ Withdrawing money is free when the cardholder uses an ATM belonging to his or her own bank. For the Reserve Bank of Australia, the objective of the "direct charging reform" was to encourage ATM deployment, promote competition for ATM services, and improve price transparency. ${ }^{2}$ In the RBA bulletin of June 2010, Reform of the ATM System - One Year On, Filipovski and Flood (2010) note that the reform contributed to a 6 percent increase in the number of ATMs between March 2009 and March 2010, but admit that the price of a typical foreign ATM transaction did not decrease. ${ }^{3}$ Second of all, as of June 2010, independent ATM deployers (IADs) owned about half of the approximately 28,800 ATMs, while banks and credit unions owned the remainder. IADs have also been responsible for half of the deployment surge since the reform. They only offer ATM services and have been present in the withdrawal market since 1997. According to the RBA, their existence has permitted the extension of ATM services to places that might otherwise not have been covered.

The ATM literature includes several works on the impact of a direct charging reform. This literature shows that such a reform increases ATM deployment (Donze and Dubec, 2009) at the cost of higher ATM usage fees (Massoud and Bernhardt, 2002). The reason is as follows: ATM fees introduce some incompatibility between banks' ATM networks. The first consequence of this incompatibility is that a bank can expand its deposit market share by raising its ATM fees, as this increases the cost for customers of banking with a competitor. Banks take this into account, and choose ATM fees above the

\footnotetext{
${ }^{1}$ In the previous system, each foreign withdrawal incurred two fees: an interchange fee flowing from the issuing bank to the acquirer (the ATM owner) and a foreign fee paid by the cardholder to his or her own bank.

${ }^{2}$ Salop (1990) designed the direct charging scheme to eliminate interchange fees and enhance the self-regulation of the withdrawal market. The collusive role of the interchange fee is analyzed theoretically by Matutes and Padilla (1994) and Donze and Dubec (2006) \& (2009).

${ }^{3}$ In the RBA bulletin of March 2011, Flood, Hancock, and Smith (2011) even report a slight increase of ATM fees between May 2010 and December 2010.
} 
level they would choose if they considered the withdrawal market separately from the deposit market. The second consequence is that a customer prefers to be a cardholder of a bank with many ATMs, in order to limit the number of foreign withdrawals. Banks are therefore incentivized to deploy ATMs in order to attract depositors. This effect is strengthened by the high ATM fees banks earn from foreign withdrawals. Banks suffer from direct charging: their profits are negatively affected by the deployment race. Consumers benefit from direct charging when they have a strong preference for large ATM networks. In this case, they take advantage of the deployment surge even if withdrawals are more expensive.

The previous papers do not take into account the existence of independent deployers of ATMs. By competing with banks in deployment and prices, IADs affect the welfare of both consumers and banks. The objective of this paper is to study the impact of direct charging when both banks and IADs are present in the withdrawal market. Do IADs benefit consumers by enlarging the ATM network or by making foreign withdrawals less expensive? Do they diminish bank profitability by intensifying the competition for withdrawals?

We show that IAD entry favors ATM deployment. Paradoxically, banks benefit from IAD entry. The intuition is the following. Under direct charging, a bank can attract depositors by expanding its ATM network. This effect is weakened when IADs enter the market: as their ATMs are accessible to all cardholders at the same price, banks become less differentiated by their own networks. Consequently, banks deploy fewer machines and their profits increase. We also show that consumer surplus decreases when the first independent deployers enter the market: IAD entry makes banks deploy fewer ATMs, so it becomes increasingly difficult for cardholders to find a free ATM. As more IADs enter, banks exit the ATM business altogether and consumer surplus increases with ATM deployment. However, consumer surplus only recovers its initial level if consumers value the enlargement of the ATM network very highly.

Several other papers consider the competition between banks and independent deployers on the withdrawal market. In Donze and Dubec (2010), we study the effects of a cost-based regulation of the interchange fee - an alternative reform which is also intended to limit its collusive power - on ATM deployment. ATM services are then provided by banks and a fixed number of independent deployers. We consider the pricing scheme used in Great Britain: banks do not charge for ATM usage but receive an interchange fee on shared 
transactions, while IADs do not receive interchange fees but charge consumers direct ATM fees. We show that such cost-based regulation causes the interchange fee to fall over time. This progressively reduces banks' incentives to deploy free ATMs and pushes IADs to deploy pay-to-use machines. Wenzel (2010) also considers the pricing scheme used in Great Britain and studies the impact of IAD entry on welfare for different levels of the interchange fee. We will compare his results with those of the present paper.

The paper is organized as follows. In section 2, we set up the model. In section 3 , we consider the case in which there are no IADs. In section 4 , we consider the case with banks and IADs. Section 5 concludes.

\section{The model}

There are two banks denoted by $i \in\{1,2\}$ located at the two ends of a product space $[0,1]$. A mass one of consumers of banking services are distributed uniformly along the product space. There are $d$ independent ATM deployers denoted by $k \in\{1, \ldots, d\}$.

\section{Banks and IADS}

Bank $i$ provides its cardholders with basic banking services (deposits) and free access to its $n_{i}$ ATMs in exchange of an account fee $p_{i}$. The marginal cost of providing the basic services is normalized to zero. IADs do not have cardholders and only provide ATM services. The number of ATMs operated by IAD $k$ is denoted by $\widehat{n}_{k}$. The total number of ATMs is $n=n_{1}+n_{2}+\sum_{k=1}^{k=d} \widehat{n}_{k}$. The cost to a bank of deploying and operating an ATM is $c{ }^{4}$ We introduce a cost difference between banks and IADs: the cost to an IAD of deploying and running an ATM is $\mu c$ where $\mu$ is an exogenous parameter satisfying $0<\mu<3 / 4 .^{5}$ The marginal cost of processing a withdrawal is normalized to zero.

\footnotetext{
${ }^{4}$ This cost includes installation, depreciation, site rental, maintenance, communication costs, cash replenishment, and the opportunity cost of the cash in the machine.

${ }^{5}$ Empirical evidence available for the USA and the UK suggests that $\mu=0.5$ is a reasonable value. In the UK, the typical cost to a bank of operating an on-premise ATM is $£ 19,000$ per year, and $£ 33,000$ for an off-premise ATM. The same service costs $£ 9,500$ to an IAD (House of Commons, Treasury Committee. 2005). In the USA, according to the 2006 ATM deployer report (Dove Consulting, 2006), a large bank incurs annual operating costs of $\$ 13,572$ for an on-premise ATM and $\$ 20,832$ for an off-premise ATM. The annual operating cost is $\$ 8,160$ per ATM for a large IAD.
} 
We consider the following pricing scheme ("direct charging"):

- There is no interchange fee.

- Bank $i$ does not charge its own cardholders for ATM usage.

- Bank $i$ 's cardholders pay a fee $s_{j}$ to bank $j$ for each withdrawal made at an ATM belonging to $j$.

- IAD $k$ charges all cardholders a fee $\widehat{s}_{k}$ per withdrawal made at its machines.

In other words, each bank discriminates between its own and its competitor's cardholders for ATM usage. On the contrary, IADs charge the same fee to everyone.

\section{Consumers}

Consumers' reservation utility is zero. A customer who becomes a depositor/cardholder of bank $i$, located at a distance $\delta_{i}$ in the product space, anticipates a surplus equal to:

$$
C S_{i}=v_{b}-t \delta_{i}+v_{i}-p_{i}
$$

The term $v_{b}$ represents the fixed surplus from consuming basic banking services. The term $t \delta_{i}$ is a differentiation cost in the product space. To guarantee the existence of a solution, $t$ must be sufficiently large, and we must have $v_{b} \geq \frac{3}{2} t$. The term $v_{i}$ corresponds to the variable net surplus from consuming withdrawals. More precisely,

$$
v_{i}=u_{i}-s_{j} q_{i}^{j}-\sum_{k=1}^{d} \widehat{s}_{k} \widehat{q}_{i}^{k},
$$

where $u_{i}$ is the variable gross surplus from consuming withdrawals, $q_{i}^{i}$ is the number of domestic withdrawals made by a cardholder of bank $i, q_{i}^{j}$ is the number of withdrawals made at bank $j$ 's ATMs (with $j \neq i$ ), and $\widehat{q}_{i}^{k}$ is the number of withdrawals made at IAD $k$ 's ATMs.

To construct the surpluses $u_{i}$ and $v_{i}$ and the demands for withdrawals, we follow Donze and Dubec (2006 \& 2009) and Chioveanu, Fauli-Oller, Sandonís, and Santamaría (2009). Any cardholder of bank $i$ faces $w n^{\gamma}$ needs of withdrawing cash, with $0 \leq \gamma<1$. Cardholders are willing to make more 
withdrawals as the network grows because it is easier to find an ATM. We will see that the higher $\gamma$, the more consumers value an enlargement of the total network. When a cardholder is looking for cash, the probability of finding an ATM belonging to a particular deployer (bank or IAD) is equal to the ATM market share of this deployer. Once an ATM has been found, any further search is infinitely costly. The consumer's valuation of this withdrawal is $r$ where $r$ is a random draw following a uniform law over $[0,1]$. As a consequence the withdrawal occurs with probability one if the ATM belongs to $i$. It is made with probability $\operatorname{Pr}\left(s_{j} \leq r\right)=1-s_{j}$ (respectively $\operatorname{Pr}\left(\widehat{s}_{k} \leq r\right)=1-\widehat{s}_{k}$ ) if the ATM belongs to bank $j$ (respectively to IAD $k$ ). Combining equations, we obtain the individual demands for withdrawals. A cardholder of bank $i$ makes $q_{i}^{i}$ withdrawals at $i$ 's ATMs, with

$$
q_{i}^{i}=w \frac{n_{i}}{n} n^{\gamma}
$$

$q_{i}^{j}$ withdrawals at bank $j$ 's machines, with:

$$
q_{i}^{j}=w\left(1-s_{j}\right) \frac{n_{j}}{n} n^{\gamma}
$$

$\widehat{q}_{i}^{k}$ withdrawals at IAD $k$ 's machines, with:

$$
\widehat{q}_{i}^{k}=w\left(1-\widehat{s}_{k}\right) \frac{\widehat{n}_{k}}{n} n^{\gamma} .
$$

Note that the cardholders of the two banks have the same demand for withdrawals at k's ATMs. Hence, we can drop subscript $i$ in $\widehat{q}_{i}^{k}$.

In appendix 1 , we determine a variable net surplus $v_{i}$ consistent with the demands given by equations (3), (4) and (5). We obtain:

$$
v_{i}=\frac{w}{2} n^{\gamma}\left(\frac{n_{i}}{n}+\frac{n_{j}}{n}\left(1-s_{j}\right)^{2}+\sum_{k=1}^{k=d} \frac{\widehat{n}_{k}}{n}\left(1-\widehat{s}_{k}\right)^{2}\right) .
$$

Consumer variable net surplus depends on the ATM market shares of the different deployers negatively weighted by the ATM fees. For given market shares, it also depends on the total network size: when banks and IADs increase the size of their respective ATM networks by a factor $\lambda>1$, then $v_{i}$ is increased by a factor $\lambda^{\gamma}$. Hence, a higher $\gamma$ means that consumers value an enlargement of the total network more highly; they are less quickly satiated by extra ATMs. 


\section{Demands and profits}

The assumptions on $v_{b}$ and $t$ ensure that the market for deposits is entirely covered. Let $\delta$ denote the distance between bank 1 and the consumer who is equally well-off when purchasing services from bank 1 or bank 2 :

$$
v_{1}-t \delta-p_{1}=v_{2}-t(1-\delta)-p_{2} .
$$

We thus obtain the size of the deposit market of bank $i$ :

$$
D_{i}=\frac{1}{2}+\frac{1}{2 t}\left(v_{i}-v_{j}-p_{i}+p_{j}\right) .
$$

IADs do not compete with banks in the market for deposits and provide exactly the same withdrawal services to all cardholders: their existence does not affect consumers' decision about where to bank. The profit of bank $i$ can be written as

$$
\pi_{i}=p_{i} D_{i}+s_{i} q_{j}^{i}\left(1-D_{i}\right)-c n_{i} .
$$

The first part of the profit corresponds to the revenue from selling basic banking services. The second part corresponds to the revenue from the withdrawals that bank $j$ 's cardholders make at bank $i$ 's machines. The third part is the cost of deploying and operating the ATMs. The profit of IAD $k$ is

$$
\widehat{\pi}_{k}=\widehat{s}_{k} \widehat{q}^{k}-\mu c \widehat{n}_{k} .
$$

In this expression, revenue comes from a mass one of cardholders, each making $\widehat{q}^{k}$ withdrawals at $k$ 's ATMs.

\section{Timing of the game}

First, banks and IADs choose the number of ATMs they deploy and choose their prices non-cooperatively and simultaneously. Second, consumers choose their banks and withdraw cash. 


\section{The case without independent ATM deploy- ers}

Without IADs, we have $d=0$. To characterize the Nash equilibrium of the model, it is convenient to start by determining the account fee. Setting $\partial \pi_{i} / \partial p_{i}=0$, and by symmetry with bank $j$, we obtain

$$
p_{i}^{*}=t+s_{i}^{*} q_{j}^{i *} .
$$

The account fee is the sum of the differentiation parameter and the cost for bank $i$ of accepting an extra depositor. The latter is actually an opportunity cost corresponding to the revenues that bank $i$ would obtain if the consumer chose to become a cardholder of bank $j$ and made $q_{j}^{i}$ withdrawals at $i$ 's ATMs. Let us now determine ATM fees. The first order condition is $\partial \pi_{i} / \partial s_{i}=0$, or:

$$
\left(p_{i}-s_{i} q_{j}^{i}\right) \frac{\partial D_{i}}{\partial s_{i}}+\left(s_{i} \frac{\partial q_{j}^{i}}{\partial s_{i}}+q_{j}^{i}\right)\left(1-D_{i}\right)=0 .
$$

By increasing $s_{i}$, bank $i$ makes it less interesting for consumers to join bank $j$, and so bank $i$ 's deposit market share increases: the first term of (12) measures how $i$ 's revenue is affected by the new depositors. The second term represents the effect of modifying $s_{i}$ on the revenue coming from foreign withdrawals. In appendix 2 , we solve equation (12) and obtain $s_{i}^{*}=2 / 3$. To attract depositors, bank $i$ chooses an ATM fee above the level it would choose if it considered ATMs as a stand-alone business: $s_{i}^{*}=2 / 3>1 / 2=\arg \max _{s_{i}} s_{i} q_{j}^{i}$.

Finally, let us determine equilibrium deployment. We have $\partial \pi_{i} / \partial n_{i}=0$, or:

$$
\left(p_{i}-s_{i} q_{j}^{i}\right) \frac{\partial D_{i}}{\partial n_{i}}+s_{i} \frac{\partial q_{j}^{i}}{\partial n_{i}}\left(1-D_{i}\right)=c .
$$

By installing a supplementary ATM, bank $i$ attracts extra depositors: the first term of (13) shows how its revenue is affected by these newcomers. Because of the supplementary ATM, bank $j$ 's cardholders also make more foreign withdrawals: the second term of (13) measures the corresponding extra revenues for bank $i$. In appendix 2, we show that, at equilibrium,

$$
\frac{\partial D_{i}}{\partial n_{i}}=\frac{2}{9 t} \frac{w}{n^{1-\gamma}}
$$

Expression (14) is decreasing in $n$ which means that it becomes increasingly difficult for banks to attract cardholders by deploying ATMs as the total network becomes larger. This stems from the fact that the value to consumers 
of an extra ATM decreases with the network size. This effect will play an important role in the next section. In appendix 2, we solve expression (13) and obtain

$$
n^{*}=\left(\frac{5+\gamma}{18} \frac{w}{c}\right)^{\frac{1}{1-\gamma}} \text {. }
$$

The surplus of the indifferent consumer (net of $v_{b}-\frac{3 t}{2}$ ) is $C S=w n^{\gamma} / 6$. Banks' total profits (net of $t$ ) are $B S=-(1+\gamma) w n^{\gamma} / 18<0$. The total surplus is $T S=C S+B S=(2-\gamma) w n^{\gamma} / 18$. The results are summarized in the following proposition:

Proposition 1 Under direct charging, bank decisions on the withdrawal market affect the deposit market. Banks overinvest in ATMs to attract depositors: their profits are smaller than they would be if banks did not provide ATM services at all (that is 0 , net of $t$ ). Banks also set ATM usage fees above the level they would choose if they considered the withdrawal market as a stand-alone business.

The regulator is likely to underestimate the spillover effects between markets when evaluating the relevance of the ATM direct charging scheme. For example, on average, banks lose money on their ATM operations in the USA. ${ }^{6}$ According to Dove Consulting (2006), the average monthly revenue per onpremise ATM was $\$ 1,104$ while expenses were $\$ 1,444$. Furthermore, according to estimates made by Knittel and Stango (2011), large banks' surcharges are $71 \%$ higher than they would be if the withdrawal market was considered as a stand-alone business. In the next section, we study how IAD entry alters the previous findings.

\section{Effects of IAD entry on bank profitability and consumer surplus}

We now assume that IADs are present in the withdrawal market: $d>0$. We first determine the equilibrium for a given number of independent deployers, and then study how welfare is affected as more IADs enter the market.

\footnotetext{
${ }^{6}$ Although the American ATM pricing scheme (interchange fee, foreign fees and surcharges) and the new Australian pricing scheme are not the same, they are formally equivalent according to the so-called neutrality result (Salop (1990), Croft and Spencer (2004), Donze and Dubec (2009), Chioveanu, Fauli-Oller, Sandonis and Santamaria (2009)).
} 


\subsection{Taxonomy of equilibria for a given number of IADs}

We solve for the Nash equilibrium. Details of the derivations are relegated to appendix 3. Bank $i$ 's maximization problem gives the same expressions as before: equations (12) and (13). ${ }^{7}$ It follows that at equilibrium, the account fee and the ATM fee remain $p_{i}^{*}=t+s_{i}^{*} q_{j}^{i *}$ and $s_{i}^{*}=2 / 3$. The ATM fee is not affected by IAD entry because according to our framework, the willingness to pay for a withdrawal is independent from deployment. We next solve the maximization problem of IAD $k$. We start by determining the ATM fees. The first order condition, $\partial \widehat{\pi}_{k} / \partial \widehat{s}_{k}=0$, gives $\widehat{s}_{k}^{*}=1 / 2$. Note that $s_{i}^{*}>\widehat{s}_{k}^{*}$ : IADs choose lower ATM fees than banks because they are not active in the deposit market and therefore do not use ATM pricing strategically to attract depositors. Let us finally consider the deployment problem. The first order condition for an interior solution, $\partial \widehat{\pi}_{k} / \partial \widehat{n_{k}}=0$, can be written as:

$$
\widehat{s}_{k} \frac{\partial \widehat{q}_{k}}{\partial \widehat{n}_{k}}=\mu c .
$$

Comparing expression (16) with expression (13) indicates that IAD $k$ does not face the same incentives to deploy as bank $i$. There is one factor pushing IAD $k$ to deploy fewer ATMs than a bank: IAD $k$ does not deploy ATMs to influence the deposit market. There are two factors pushing IAD $k$ to deploy more ATMs than a bank. First, IAD $k$ charges all cardholders for their withdrawals, while bank $i$ only charges non-customers. Second, $k$ has a cost advantage over banks because $\mu<3 / 4$. In appendix 3 , we verify that there are two types of equilibria according to the value of $\mu$. They are detailed in the following proposition:

Proposition 2 Consider a given $d \geq 1$. There are two types of equilibria, depending on the value of $\mu$ :

- If $\frac{3}{4} \frac{d-1+\gamma}{d}<\mu<\frac{3}{4}$, both banks and IADs deploy ATMs: $n^{*}=\left(\frac{5+d+\gamma}{18+4 \mu d} \frac{w}{c}\right)^{\frac{1}{1-\gamma}}, \frac{n_{i}^{*}}{n^{*}}=\frac{9(1-\gamma)+(12 \mu-9) d}{(1-\gamma)(18+4 \mu d)}, \frac{\widehat{n}_{k}^{*}}{n^{*}}=\frac{9-10 \mu-2 \gamma \mu}{(1-\gamma)(9+2 \mu d)}$.

- If $\mu \leq \frac{3}{4} \frac{d-1+\gamma}{d}$, only IADs deploy ATMs: $n^{*}=\left(\frac{1}{4} \frac{d-1+\gamma}{d} \frac{w}{\mu c}\right)^{\frac{1}{1-\gamma}}, \frac{\widehat{n}_{k}^{*}}{n^{*}}=\frac{1}{d}$.

\footnotetext{
${ }^{7}$ Assuming an interior solution; otherwise, condition (13) should be written with a sign $\leq$. See appendix 3 for details.
} 
When $\mu$ is high, both banks and IADs deploy ATMs. In this case the total network size and the ATM market share of IADs increase as their cost advantage over banks becomes higher (i.e. when $\mu$ decreases). When $\mu$ is low, banks do not deploy ATMs and they only produce basic banking services.

\subsection{Effects of IAD entry on profits and consumer sur- plus}

We now study how consumer surplus, banks' and IADs' profits and total surplus are modified as the number of IADs increases, starting from $d=0 .{ }^{8}$ Let us define $\widetilde{d}$ by

$$
\widetilde{d}=\frac{3(1-\gamma)}{3-4 \mu}
$$

When $d$ is equal to zero, only banks deploy ATMs. When $d$ is between 1 and $\widetilde{d}$, banks and IADs deploy ATMs. When $d$ is larger than $\widetilde{d}$, only IADs deploy ATMs. In what follows, the surplus of the indifferent consumer is written net of $v_{b}-\frac{3 t}{2}$. Banks' total profits are also written net of $t$. The results are established in appendix 4.

Deployment. As independent deployers enter the market, the total number of ATMs rises. Banks' ATM market share decreases and becomes nil as soon as $d$ reaches $\widetilde{d}$. Banks deploy fewer and fewer ATMs for two reasons. First, IADs compete with them for foreign withdrawals, diminishing their incentives to deploy ATMs (in the second term of expression (13), $\partial q_{j}^{i} / \partial n_{i}$ is decreasing in $n$ ). Second IAD entry makes ATMs a less important differentiator for banks: at equilibrium,

$$
\frac{\partial D_{i}}{\partial n_{i}}=\frac{2}{9 t} \frac{w}{n^{1-\gamma}}
$$

is decreasing in $n$ and banks are less willing to deploy ATMs to attract depositors. $^{9}$

Prices. The account fee decreases with IAD entry from $d=0$ to $d=\widetilde{d}$ and remains constant thereafter. Indeed, $p_{i}^{*}$ is the sum of the differentiation parameter $t$ and the opportunity cost of accepting a new cardholder (the foregone

\footnotetext{
${ }^{8}$ We treat $d$ as if it was a continuous variable. Results on surplus variations may be locally different from those obtained with a discrete number of IADs.

${ }^{9}$ In the model, we ignore one further reason for which banks deploy ATMs: as a substitute for more costly human tellers. If this was included in the model, banks would probably keep their on-premise ATMs despite IAD entry, but would abandon their off-premise ATMs.
} 
ATM fees this cardholder would have paid to bank $i$ if he had chosen to bank with $j$ ). This opportunity cost decreases as IADs enter the market and deploy ATMs. It becomes nil when banks exit the withdrawal market, that is, when $d$ is larger than $\widetilde{d}$.

Consumer surplus. When $d$ is below $\widetilde{d}$, consumer surplus is equal to

$$
C S(d)=\frac{1}{8} w \frac{24-6 d+12 \mu d-4 \gamma(6+\mu d)}{(1-\gamma)(18+4 \mu d)}\left(\frac{5+d+\gamma}{18+4 \mu d} \frac{w}{c}\right)^{\frac{\gamma}{1-\gamma}}
$$

which is decreasing in $d$. IAD entry affects consumer surplus in several ways. There is one negative effect: it is more difficult for bank $i$ 's cardholders to find a free ATM, because bank $i$ 's ATM market share diminishes. There are three positive effects. First, there are more ATMs available from which to withdraw money. Second, on average, foreign withdrawals become cheaper since they are increasingly made at IAD ATMs and less frequently at bank j's ATMs. Third, account fees also decrease, as explained above. Overall, the negative effect outweighs the positive effects and consumer surplus decreases when the first IADs enter the market. When $d$ is larger than $\widetilde{d}$, banks exit the withdrawal market. Consumer surplus becomes

$$
C S(d)=\frac{1}{8} w\left(\frac{1}{4} \frac{d-1+\gamma}{d} \frac{w}{\mu c}\right)^{\frac{\gamma}{1-\gamma}}
$$

which increases with IAD entry if consumers value the enlargement of the ATM network (that is, if $\gamma>0$ ). As banks are no longer active in the withdrawal market, IAD entry has only a positive effect on consumer surplus through the enlargement of the ATM network.

In addition to the previous incremental analysis, it is interesting to compare consumer surplus under entry to the case without IADs. When $\gamma$ is large enough, there is a level of entry, $\bar{d}(\gamma, \mu) \geq \widetilde{d}$, such that for any $d \geq \bar{d}(\gamma, \mu)$, consumer surplus, $C S(d)$ defined in equation (20), is higher than its initial level, $C S(0) .{ }^{10}$ In other words, when consumers have strong preferences for large ATM networks, an entry of a large number of IADs benefits consumers. Note that in this case, banks are not active in the ATM business. For example, $\gamma$ must be higher than 0.357 when $\mu=0.5$.

\footnotetext{
${ }^{10}$ One can easily verify that $\lim _{d \rightarrow \infty} C S(d)>C S(0)$ when $\gamma$ is close to 1 . This guarantees the existence of the threshold, $\bar{d}(\gamma, \mu)$.
} 
Banks' profits. When $d$ is below $\widetilde{d}$, banks' profits are equal to

$B S(d)=-\frac{2 w}{3} \frac{[3(1-\gamma)+(4 \mu-3) d][9+9 \gamma+(9-8 \mu) d]}{(1-\gamma)(18+4 \mu d)^{2}}\left(\frac{5+d+\gamma}{18+4 \mu d} \frac{w}{c}\right)^{\frac{\gamma}{1-\gamma}}$

and are increasing in $d$. IAD entry causes banks to deploy fewer and fewer ATMs, but also reduces account and ATM fee revenues. The positive effect due to deployment cost savings is stronger than the negative effects on revenues and banks' profits actually increase with IAD entry. When $d$ is above $\widetilde{d}$, banks' profits (net of $t$ ) are constant and equal to 0 .

IADs' profits. When $d$ is below $\widetilde{d}$, IADs' profits are equal to

$$
I A D S(d)=w \frac{(9-10 \mu-2 \gamma \mu)^{2} d}{(1-\gamma)(18+4 \mu d)^{2}}\left(\frac{5+d+\gamma}{18+4 \mu d} \frac{w}{c}\right)^{\frac{\gamma}{1-\gamma}} .
$$

When $\mu \leq 9 / 14+3 \gamma / 28$, expression (22) is increasing in $d$ : the first independent deployers that enter the market replace banks in the ATM business and make increasing profits. When $\mu>9 / 14+3 \gamma / 28$, expression (22) is first increasing and then decreasing with entry. In this case, the cost advantage of IADs over banks is weaker, and IADs' profits start to decrease before banks exit the withdrawal market. When $d$ is above $\widetilde{d}$, IADs' profits are equal to

$$
\operatorname{IADS}(d)=w \frac{1-\gamma}{4 d}\left(\frac{1}{4} \frac{d-1+\gamma}{d} \frac{w}{\mu c}\right)^{\frac{\gamma}{1-\gamma}}
$$

and are decreasing with entry: more and more independent deployers chase a number of potential withdrawals, $w n^{\gamma}$, with an ever-increasing number of ATMs, $n$.

Total surplus. Total surplus is given by $T S=C S+B S+I A D S$. It is increasing with entry when $d$ is below $\widetilde{d}$. Initially, total surplus follows the rise in banks' and IADs' profits. Furthermore, banks' ATMs are replaced by IADs' less costly machines. When $d$ is above $\widetilde{d}$, three cases are possible. If $\gamma \leq 8 \mu / 9$, total surplus decreases with entry. In this case, the rise in consumer surplus does not compensate for the fall in IADs' profits. If $8 \mu / 9<\gamma<2 / 3$, total surplus first increases and then decreases with entry. At first, total surplus follows the increase in consumer surplus, but this is later outweighed by the fall in IADs' profits. If $\gamma \geq 2 / 3$, total surplus is monotonically increasing with entry. 
It is interesting to compare total surplus under entry to the case without IADs. One can verify that for any $\gamma$ and any entry level, total surplus is higher in the presence of IADs than under the no-entry case. Hence, if we take the unweighted sum of surpluses as a measure of social welfare, IAD entry is socially good. The main reason for this result is that IAD entry benefits banks by limiting the deployment race. For high values of $\gamma$, the positive impact of entry on consumer surplus reinforces the previous effect. The main results are summarized in proposition 3.

Proposition 3 IAD entry makes it less interesting for each bank to use ATMs as a way to differentiate from its competitor and attract depositors. As a consequence, the two banks deploy fewer ATMs and their profits increase. Consumer surplus initially decreases with IAD entry, as it becomes more difficult to make a free withdrawal. Subsequently, consumer surplus increases as more ATMs are deployed, but only retrieves its initial level if consumers place a high value on large ATM networks.

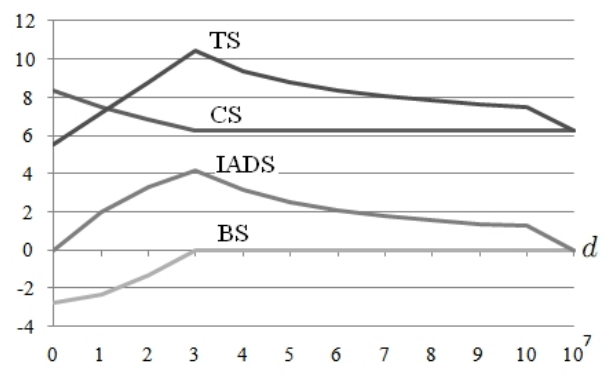

(a) $\mu=0.5, \gamma=0, w=50$

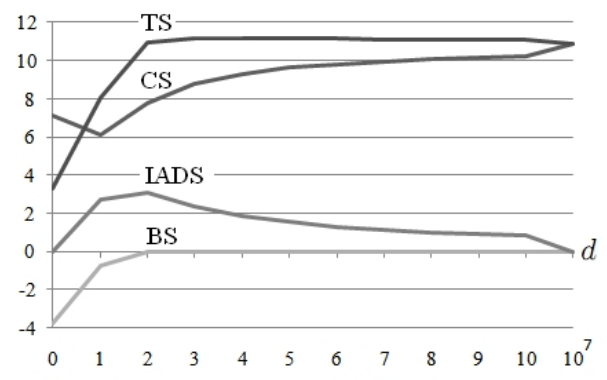

(c) $\mu=0.5, \gamma=0.6, w=2900$


(d) $\mu=0.7, \gamma=0, w=50$

Figure 1: Changes in surpluses with IAD entry

To illustrate the changes in surplus resulting from IAD entry, we have drawn $C S, B S, I A D S$ and $T S$ in figure 1 for $c=15000$ and different values of 
$\mu, \gamma$ and $w .^{11}$ Figures $1(a),(b)$ and (c) are drawn for $\mu=0.5$ and show how increasing $\gamma$ affects the change in consumer surplus. Consumer surplus decreases with entry as long as banks are active in the withdrawal market but thereafter increases if consumers value the enlargement of the network. The increase is more pronounced when $\gamma$ is high. Consider, for example, figure 1(c) where $\gamma=0.6$. In this case, for a sufficient level of entry, consumers are better off compared to the situation where ATM services are provided exclusively by banks. Figure 1 (c) also verifies $8 \mu / 9<\gamma<2 / 3$ : total surplus reaches its maximum for a number of IADs higher than $\widetilde{d}$. In this case, the change in total surplus follows the change in consumer surplus up to some level of entry at which banks have already left the withdrawal market. Figure 1(d) illustrates the case where $\mu>9 / 14+3 \gamma / 28$. In this case, IADs' profits decrease whereas banks are still active in the withdrawal market.

It is interesting to compare the effects of IAD entry under the direct charging scheme and an interchange-based scheme. Wenzel (2010) considers a case where (i) banks do not charge consumers for ATM usage but receive an interchange fee on shared transactions (ii) IADs do not receive interchange fees but charge consumers direct ATM fees. He obtains similar results to ours regarding the effect of IAD entry on deployment, account fees and consumer surplus: entry favors ATM deployment and lowers account fees. Furthermore, the range of interchange fees for which consumers benefit from IAD entry becomes larger as consumer preferences for large ATM networks grow. Nevertheless, the effect of entry on banks' profits is very different in the framework considered by Wenzel. As banks do not charge for ATM usage, their ATM networks are perfectly equivalent for cardholders, no matter their affiliation. As a result, there is no deployment race to attract depositors and IAD entry induces lower deployment cost savings for banks than under direct charging. In this case, entry reduces bank profits by diminishing account fees and strengthening the competition for withdrawals.

\footnotetext{
${ }^{11}$ We calibrate the model for different values of $\mu$ and $\gamma$ by choosing $w$ so that, at equilibrium, total ATM deployment remains close to 1/1000. The calibration permits to obtain similar scales when we represent the surpluses in the different cases.
} 


\section{Concluding remarks}

On the 3rd of March 2009, the Reserve Bank of Australia suppressed interchange fees on shared ATM transactions and replaced them by direct fees charged by ATM owners. The objective was to design a competitive withdrawal market. The Australian reform could be imitated in other countries and it is therefore important to assess its implications, both theoretically and empirically. In Donze and Dubec (2009), we showed that ATM direct charging boosts deployment and makes consumers better off than they would be under a regime with interchange and foreign fees, as long as consumers have a strong preference for large networks (which seems reasonable when travel costs are high). However, direct charging negatively affects bank profitability. In the present article we have shown that the entry of independent deployers limits banks' incentives to use ATM deployment strategically to expand their deposit market shares. This mitigates the deployment race between banks, and their profits increase. The effect of IAD entry on consumer surplus is ambiguous. As long as banks are active in the withdrawal market, consumer surplus decreases with IAD entry. Once banks abandon the ATM business, consumer surplus increases with entry. However, consumer surplus can only recover its no-entry level if consumers have a strong preference for large ATM networks.

\section{Appendices}

Appendix 1. Expression of $u_{i}$ and $v_{i}$.

Let us consider $v_{i}=u_{i}-s_{j} q_{i}^{j}-\sum_{k=1}^{d} \widehat{s}_{k} \widehat{q}_{i}^{k}$ with

$$
u_{i}=\left(q_{i}^{i}-\frac{n^{1-\gamma}}{2 w n_{i}}\left(q_{i}^{i}\right)^{2}\right)+\left(q_{i}^{j}-\frac{n^{1-\gamma}}{2 w n_{j}}\left(q_{i}^{j}\right)^{2}\right)+\sum_{k=1}^{d}\left(\widehat{q}_{i}^{k}-\frac{n^{1-\gamma}}{2 w \widehat{n}_{k}}\left(\widehat{q}_{i}^{k}\right)^{2}\right) .
$$

Differentiating $v_{i}$ with respect to $q_{i}^{i}, q_{i}^{j}$ and $\widehat{q}_{i}^{k}$ we obtain the demand functions (3), (4) and (5). Plugging these three expressions in $v_{i}$, we obtain expression (6), the optimized variable net surplus from withdrawing.

Appendix 2. Proof of proposition 1

Using expressions (2) and (8) one can write

$$
\frac{\partial D_{i}}{\partial s_{i}}=-\frac{1}{2 t} \frac{\partial v_{j}}{\partial s_{i}}=-\frac{1}{2 t}\left(\frac{\partial u_{j}}{\partial q_{j}^{i}} \frac{\partial q_{j}^{i}}{\partial s_{i}}-q_{j}^{i}-s_{i} \frac{\partial q_{j}^{i}}{\partial s_{i}}\right) .
$$


However at consumer's optimum, $\partial u_{j} / \partial q_{j}^{i}=s_{i}$, so that

$$
\frac{\partial D_{i}}{\partial s_{i}}=\frac{1}{2 t} q_{j}^{i} .
$$

Using expressions (11) and (26), one can rewrite equation (12) as

$$
\frac{1}{2} q_{j}^{i}+\left(-w s_{i} \frac{n_{i}}{n} n^{\gamma}+q_{j}^{i}\right)\left(1-D_{i}\right)=0 .
$$

At equilibrium, $D_{i}=1 / 2$ and we obtain $s_{i}^{*}=2 / 3$. Let us now determine the equilibrium deployment, we have

$$
\frac{\partial D_{i}}{\partial n_{i}}=\frac{1}{2 t} \frac{\partial\left(v_{i}-v_{j}\right)}{\partial n_{i}} \underset{\text { (equilibrium) }}{=} \frac{2}{9 t} w n^{\gamma-1} .
$$

Expression (11) and (13) gives

$$
\frac{2}{9} w n^{\gamma-1}+w s_{i}\left(1-s_{i}\right)\left(1-D_{i}\right)\left(n^{\gamma-1}+n_{i}(\gamma-1) n^{\gamma-2}\right)=c .
$$

At equilibrium we have $D_{i}=1 / 2, n_{i}=n / 2, s_{i}=2 / 3$, and we obtain

$$
n^{*}=\left(\frac{5+\gamma}{18} \frac{w}{c}\right)^{\frac{1}{1-\gamma}}
$$

Appendix 3. Proof of proposition 2

The problem of maximization has two types of solutions: interior or corner. We have $\partial \pi_{i} / \partial n_{i} \leq 0$ and $\partial \widehat{\pi}_{k} / \partial \widehat{n}_{k} \leq 0$ for any $i$ and $k$ :

$$
\frac{w}{9}\left(3-(1-\gamma) \frac{n_{i}}{n}\right) n^{\gamma-1}-c \leq 0
$$

and

$$
\frac{w}{4}\left(1-(1-\gamma) \frac{\widehat{n}_{k}}{n}\right) n^{\gamma-1}-\mu c \leq 0 .
$$

We first look for (interior) solutions where the two first-order conditions are satisfied with equalities. We have

$$
\mu \frac{w}{9}\left(3-(1-\gamma) \frac{n_{i}}{n}\right)=\frac{w}{4}\left(1-(1-\gamma) \frac{\widehat{n}_{k}}{n}\right) .
$$


However $n=2 n_{i}+d \widehat{n}_{k}$ or $2 \frac{n_{i}}{n}+d \frac{\widehat{n}_{k}}{n}=1$. Plugging this last equality in (33), we obtain

$$
\frac{n_{i}^{*}}{n^{*}}=\frac{9(1-\gamma)+(12 \mu-9) d}{(1-\gamma)(18+4 \mu d)}
$$

and

$$
\frac{\widehat{n}_{k}^{*}}{n^{*}}=\frac{9-10 \mu-2 \gamma \mu}{(1-\gamma)(9+2 \mu d)}
$$

Plugging expression (34) in expression (31) we obtain

$$
n^{*}=\left(\frac{5+d+\gamma}{18+4 \mu d} \frac{w}{c}\right)^{\frac{1}{1-\gamma}}
$$

For the solution to exist, one must have $\frac{n_{i}^{*}}{n^{*}} \geq 0$ and $\frac{\widehat{n}_{k}^{*}}{\widehat{n}^{*}} \geq 0$ or equivalently $\frac{3}{4}\left(\frac{d-1+\gamma}{d}\right) \leq \mu \leq \frac{9}{10+2 \gamma}$. Note this last inequality is verified because $\frac{9}{10+2 \gamma} \geq \frac{3}{4}$. Suppose $\mu \leq \frac{3}{4}\left(\frac{d-1+\gamma}{d}\right)$, we obtain the corner solution $\frac{\widehat{n}_{k}^{*}}{\widehat{n}^{*}}=\frac{1}{d}$ and $\frac{n_{i}^{*}}{n^{*}}=0$. Condition (31) is satisfied with inequality while condition (32) is satisfied with equality, we obtain

$$
n^{*}=\left(\frac{1}{4} \frac{d-1+\gamma}{d} \frac{w}{\mu c}\right)^{\frac{1}{1-\gamma}}
$$

Appendix 4. Consumer surplus, banks' surplus, IADs' surplus and total surplus

In what follows, the surplus of the indifferent consumer is written net of $v_{b}-\frac{3 t}{2}$. Similarly banks' total profits are written net of $t$. Let us define $\widetilde{d}$, the number of IADs above which banks do not deploy any ATMs:

$$
\widetilde{d}=\frac{3(1-\gamma)}{3-4 \mu}
$$

(i) Variation of CS.

For $d \leq \widetilde{d}$, the surplus of the indifferent consumer is

$$
C S(d)=\frac{1}{8} w \frac{24-6 d+12 \mu d-4 \gamma(6+\mu d)}{(1-\gamma)(18+4 \mu d)}\left(\frac{5+d+\gamma}{18+4 \mu d} \frac{w}{c}\right)^{\frac{\gamma}{1-\gamma}}
$$

Differentiating with respect to $d$, we obtain

$$
\frac{\partial C S}{\partial d}=-\frac{w}{2} \frac{(9-10 \mu-2 \mu \gamma)\left[15-24 \gamma+9 \gamma^{2}+d\left(3-6 \gamma \mu+2 \gamma^{2} \mu\right)\right]}{(1-\gamma)^{2}(5+d+\gamma)(18+4 \mu d)^{2}}\left(\frac{5+d+\gamma}{18+4 \mu d} \frac{w}{c}\right)^{\frac{\gamma}{1-\gamma}}
$$


This expression is negative. Indeed $\mu<3 / 4$ implies $9-10 \mu-2 \mu \gamma>0$. We have also $15-24 \gamma+9 \gamma^{2}=(1-\gamma)(15-9 \gamma)>0$. Furthermore, as $\max _{\gamma \in[0,1]} \gamma(3-\gamma)=$ 2 and $\mu \leq 3 / 4$, we have $2 \mu \gamma(3-\gamma) \leq 3$ so that $3-2 \mu \gamma(3-\gamma) \geq 0$. Hence $15-24 \gamma+9 \gamma^{2}+d\left(3-6 \gamma \mu+2 \gamma^{2} \mu\right)>0$ for any $d$.

For $d \geq \tilde{d}$, consumer surplus is

$$
C S=\frac{1}{8} w\left(\frac{1}{4} \frac{d-1+\gamma}{d} \frac{w}{\mu c}\right)^{\frac{\gamma}{1-\gamma}} .
$$

It is increasing in $d$ when $\gamma>0$.

(ii) Variation of BS. For $d \leq \widetilde{d}$, banks' surplus is given by

$$
B S=-\frac{2}{3} w \frac{[3(1-\gamma)+(4 \mu-3) d][9+9 \gamma+(9-8 \mu) d]}{(1-\gamma)(18+4 \mu d)^{2}}\left(\frac{5+d+\gamma}{18+4 \mu d} \frac{w}{c}\right)^{\frac{\gamma}{1-\gamma}}
$$

The derivative with respect to $d$ is

$$
\begin{array}{r}
\frac{\partial B S}{\partial d}=\frac{4 w}{3} \frac{\left[\left(54-60 \mu-27 \gamma+32 \mu^{2} \gamma\right) d^{2}+\left(270-300 \mu-162 \gamma+228 \mu \gamma-54 \gamma^{2}\right) d-27 \gamma^{3}-216 \gamma^{2}+243 \gamma\right]}{(1-\gamma)^{2}(5+d+\gamma)(18+4 \mu d)^{3}} \\
\times(9-10 \mu-2 \mu \gamma) \times\left(\frac{5+d+\gamma}{18+4 \mu d} \frac{w}{c}\right)^{\frac{\gamma}{1-\gamma}}
\end{array}
$$

This expression is positive which means that $B S$ is increasing in $d$. To prove positivity, note first that $\mu<3 / 4$ implies $9-10 \mu-2 \mu \gamma>0$. Second $54-$ $60 \mu-27 \gamma+32 \mu^{2} \gamma$ is decreasing in $\mu$ on $[0,3 / 4]$ and equal to $9(1-\gamma)>0$ when $\mu=3 / 4$. Third $270-300 \mu-162 \gamma+228 \mu \gamma-54 \gamma^{2}$ is also decreasing in $\mu$ and equal to $45+9 \gamma-54 \gamma^{2}=9(6 \gamma+5)(1-\gamma)>0$ when $\mu=3 / 4$. Finally $-27 \gamma^{3}-216 \gamma^{2}+243 \gamma=27 \gamma(\gamma+9)(1-\gamma)>0$.

For $d \geq \widetilde{d}$, banks' surplus (net of $t$ ) becomes constant and equal to 0 .

(iii) Variation of IADS. For $d \leq \widetilde{d}$, IAD surplus is given by

$$
I A D S=w \frac{(9-10 \mu-2 \gamma \mu)^{2} d}{(1-\gamma)(18+4 \mu d)^{2}}\left(\frac{5+d+\gamma}{18+4 \mu d} \frac{w}{c}\right)^{\frac{\gamma}{1-\gamma}}
$$

Differentiating with respect to $d$ we obtain

$$
\frac{\partial I A D S}{\partial d}=w \frac{(9-10 \mu-2 \gamma \mu)^{2}\left[90-72 \gamma-18 \gamma^{2}+d(18-20 \mu-4 \mu \gamma)-4 \mu d^{2}(1-\gamma)\right]}{(1-\gamma)^{2}(5+d+\gamma)(18+4 \mu d)^{3}}\left(\frac{5+d+\gamma}{18+4 \mu d} \frac{w}{c}\right)^{\frac{\gamma}{1-\gamma}} .
$$


The derivative (45) has the sign of $90-72 \gamma-18 \gamma^{2}+d(18-20 \mu-4 \mu \gamma)-$ $4 \mu d^{2}(1-\gamma)$. This expression is positive for $d=0$. The solution of $90-72 \gamma-$ $18 \gamma^{2}+d(18-20 \mu-4 \mu \gamma)-4 \mu d^{2}(1-\gamma)=0$ is equal to $\widetilde{d}$ when $\mu=9 / 14+3 \gamma / 28$. When $\mu \leq 9 / 14+3 \gamma / 28$, expression (45) is positive between $d=0$ and $d=\widetilde{d}$. When $\mu>9 / 14+3 \gamma / 28$, expression (45) is first positive and then negative between $d=0$ and $d=\widetilde{d}$

For $d \geq \widetilde{d}$, IAD surplus is equal to

$$
I A D S=w \frac{1-\gamma}{4 d}\left(\frac{1}{4} \frac{d-1+\gamma}{d} \frac{w}{\mu c}\right)^{\frac{\gamma}{1-\gamma}},
$$

and is decreasing with $d$.

(iv) Variation of $T S$. Total surplus is $T S=C S+B S+I A D S$.

For $d \leq \widetilde{d}$, the derivative of $T S$ is

$$
\begin{gathered}
\frac{\partial T S}{\partial d}=w\left[\left(432-732 \mu-72 \mu^{2} \gamma^{2}+240 \mu^{2}+136 \mu^{2} \gamma+216 \mu \gamma-216 \gamma\right) d^{2}\right. \\
+6\left(495-790 \mu-8 \mu^{2} \gamma^{2}+80 \mu^{2} \gamma-216 \gamma+200 \mu^{2}-72 \gamma^{2}+334 \mu \gamma-36 \mu \gamma^{2}\right) d \\
\left.+4050-5400 \mu+3240 \mu \gamma-648 \gamma-3186 \gamma^{2}-216 \gamma^{3}+1944 \mu \gamma^{2}+216 \gamma^{3} \mu\right] \\
\times \frac{(9-10 \mu-2 \gamma \mu)}{(1-\gamma)^{2}(5+d+\gamma)(18+4 \mu d)^{3}}\left(\frac{5+d+\gamma}{18+4 \mu d} \frac{w}{c}\right)^{\frac{\gamma}{1-\gamma}} \cdot
\end{gathered}
$$

This expression is positive so that TS is increasing in $d$ over the specified set. To show positivity, note first that $432-732 \mu-72 \mu^{2} \gamma^{2}+240 \mu^{2}+136 \mu^{2} \gamma+$ $216 \mu \gamma-216 \gamma$ is decreasing in $\mu$ on $[0,3 / 4]$ and equal to $\frac{9}{2}(9 \gamma+4)(1-\gamma)>0$ when $\mu=3 / 4$. Second, $2970-4740 \mu+48 \mu^{2} \gamma^{2}+480 \mu^{2} \gamma-1296 \gamma+1200 \mu^{2}-$ $432 \gamma^{2}+2004 \mu \gamma-216 \mu \gamma^{2}$ is decreasing in $\mu$ on $[0,3 / 4]$ and equal to $9(63 \gamma+10) \times$ $(1-\gamma)>0$ when $\mu=3 / 4$. Third, $4050-5400 \mu+3240 \mu \gamma-648 \gamma-3186 \gamma^{2}-$ $216 \gamma^{3}+1944 \mu \gamma^{2}+216 \gamma^{3} \mu$ is decreasing in $\mu$ on $[0,3 / 4]$ and equal to $54 \gamma(1-\gamma) \times$ $(\gamma+33) \geq 0$ when $\mu=3 / 4$.

For $d \geq \widetilde{d}$, total surplus becomes

$$
T S=\frac{w}{8} \frac{d+2(1-\gamma)}{d}\left(\frac{1}{4} \frac{d-1+\gamma}{d} \frac{w}{\mu c}\right)^{\frac{\gamma}{1-\gamma}} .
$$

The derivative with respect to $d$ is

$$
\frac{\partial T S}{\partial d}=\frac{w}{8} \frac{(3 \gamma-2) d+2-2 \gamma}{d^{2}(d-1+\gamma)}\left(\frac{1}{4} \frac{d-1+\gamma}{d} \frac{w}{\mu c}\right)^{\frac{\gamma}{1-\gamma}} .
$$


When $2 / 3 \leq \gamma<1$ then $\partial T S / \partial d$ is positive so that $T S$ is increasing in $d$. Consider $\gamma<2 / 3$. Let $\widehat{d}$ denote the number of IADs that makes expression (48) equal to zero. We have

$$
\widehat{d}=\frac{2-2 \gamma}{2-3 \gamma} .
$$

If $\widehat{d}>\widetilde{d}$ (or equivalently $8 \mu / 9<\gamma<2 / 3$ ), then total surplus is increasing up to $\widehat{d}$ and decreasing thereafter. If $\widehat{d} \leq \widetilde{d}$ (or equivalently $\gamma \leq 8 \mu / 9$ ), then total surplus is decreasing when $d$ is above $\widetilde{d}$.

\section{Bibliography}

Chioveanu, I., Fauli-Oller, R., Sandonís, J., and Santamaría J., 2009. ATM Surcharges: Effects on Deployment and Welfare. Journal of Industrial Economics 57 (3) p 613-635.

Croft, E., Spencer, B., 2004. Fees and Surcharging in ATM Networks: the Role of Nonbanks and Depositor Base. Working Paper.

Donze, J., Dubec, I., 2006. The role of Interchange Fees in ATM Networks. International Journal of Industrial Organization, 24 (1), p 29-43.

Donze, J., Dubec, I., 2009. Paying for ATM usage: Good for Consumers, Bad for Banks? Journal of Industrial Economics 57 (3), p 583-612.

Donze, J., Dubec, I., 2010. The Effects of Regulating Interchange Fees at Cost on the ATM Market. Economics Letters. 107 (2), p 187-189.

Dove Consulting, 2006. 2006 ATM Deployer Study.

Filipovski, B., Flood, D., 2010. Reform of the ATM System - One Year On. RBA Bulletin. June Quarter.

Flood, D., Hancock, J. and Smith K., 2011. The ATM Reforms - New Evidence from Survey and Market Data. RBA Bulletin. March Quarter.

Knittel, C., Stango, V., 2011. Strategic Incompatibility in ATM Markets. Forthcoming in Journal of Banking and Finance.

Massoud, N., Bernhardt, D., 2002. Rip-off ATM surcharges. Rand Journal of Economics 33, p 96-115. 
Matutes, C., Padilla. A. J., 1994. Shared ATM Networks and Banking Competition. European Economic Review, 38: p 1113-38.

McAndrews, J. 2003., Automated Teller Machine Network Pricing, a Review of the Literature. The Review of Network Economics, 2 (2), p 146-158.

Reserve Bank of Australia \& the Australian Competition and Consumer Commission. 2000. Debit and Credit Card Schemes in Australia: A Study of Interchange Fees and Access Competition.

Reserve Bank of Australia. 2009. Payments System Board Annual Report.

Salop, S., 1990. Deregulating Self-Regulated Shared ATM Networks. Economics of Innovation and New Technology, 1 p 85-96.

Wenzel, T., 2010. Independent Service Operators in ATM Markets. Working Paper. 